\title{
Simulation of partial discharge within a void under square waveform applied voltage
}

\author{
H.A. Illias ${ }^{1}$, A.H.A. Bakar ${ }^{1}$, H. Mokhlis ${ }^{1}$, M.A. Tunio ${ }^{1}$, G. Chen ${ }^{2}$, P.L. Lewin ${ }^{2}$ and A.M. Ariffin ${ }^{3}$ \\ ${ }^{1}$ UMPEDAC, Electrical Engineering Department \\ Faculty of Engineering, University of Malaya \\ Kuala Lumpur, Malaysia \\ ${ }^{2}$ The Tony Davies High Voltage Laboratory \\ University of Southampton \\ Southampton, United Kingdom \\ ${ }^{3}$ College of Engineering \\ Universiti Tenaga Nasional \\ Selangor, Malaysia \\ h.illias@um.edu.my
}

\begin{abstract}
Partial discharge (PD) activity within a void in a dielectric material is influenced by many factors. One of the factors is the applied voltage waveform on the material. In this paper, a two-dimensional (2D) model of a cylindrical void in polyethylene layers has been developed using finite element analysis (FEA) software. The model was used to simulate PD activity in the void under square waveform applied voltage. The obtained simulation results were compared with the measurement results from literature. It was found that both results are within reasonable agreement but with only slight disagreement. From comparison, critical parameters from the model affecting PD activity under square waveform applied voltage were identified; they include the inception and extinction voltages and electron generation rate. This finding may increase an understanding of PD behaviour within a void in a dielectric material under square waveform applied voltage.
\end{abstract}

\section{INTRODUCTION}

One of the factors which influences partial discharge (PD) activity within a void in a dielectric material is the applied voltage waveform. It was demonstrated in one of the previous literatures that PD behaviour is clearly different under $\mathrm{AC}$ sinusoidal and square waveform applied voltages [1]. Although the transition of PD amplitude with aging shows similar behaviour between AC sinusoidal and square waveform, the experiment results demonstrated that some distinctive characteristics of PDs appears under square waveform. The measurement results were explained by a simulation model of PD, which indicated there is an effect of discharge area within the void and the void surface condition change with the repetition of PD events.

An experiment of measuring PD extinction voltage under semi-square voltages of 2 us and 100 us rise times was performed using three different types of test objects [2]. The test objects were a needle for corona discharges, a twisted pair test object used to model motor insulation testing and a paper/oil test object to model the turn-to-turn insulation of a transformer winding. The extinction voltage was found to be between $1 \mathrm{kV}$ and $8 \mathrm{kV}$. At shorter rise time, the PD extinction voltage was lower for the twisted pair test objects compared to other test objects. The work concluded that the rise time of the applied semi-square waveform and the insulation system influence the PD extinction voltage.

The influence of voltage waveforms other than sinusoidal applied voltage on PD formation in high voltage insulation system, the rise time of PD signal and the development of PD was reported in [3]. The samples used were polymer thermosetting samples. The work summarized that the influence of high frequency applied voltage on the insulation materials requires investigations of PD mechanism at fast changing stimulus and new diagnostic method.

In this work, PD behaviour within a cylindrical void in polyethylene (PE) layers under square waveform applied voltage was studied. A PD model was developed using finite element analysis (FEA) software and used to simulate PD activity in the void. The simulation results were compared with the measurement results obtained in literature [1]. Through comparison, important parameters affecting PD behaviour under square waveform applied voltage can be identified clearly; they include the inception and extinction voltages and electron generation rate.

\section{EXPERIMENT SETUP}

The experiment setup is based on the work that has been performed in [1]. The test object consists of the CIGRE Method-II electrode sample, as shown in Fig. 1. The diameter of the ball electrode, which was connected to a $200 \mathrm{~Hz}$ square waveform applied voltage is $5.6 \mathrm{~mm}$. Three polyethylene (PE) layers were pressed against each other, where the middle layer consists of a cylindrical void of diameter $12 \mathrm{~mm}$. Each PE layer thickness is $200 \mathrm{um}$. The bottom electrode was always grounded. The applied voltage amplitude was varied at 3.5, 4, 5 and $6 \mathrm{kV}$.

The test object was connected to a standard PD measurement system to capture PD occurrences in the test object, 100 minutes after the applied voltage was applied [1]. Phase resolved partial discharge patterns (PRPD) were used to observe PD behaviour under square waveform applied voltage within several cycles. 


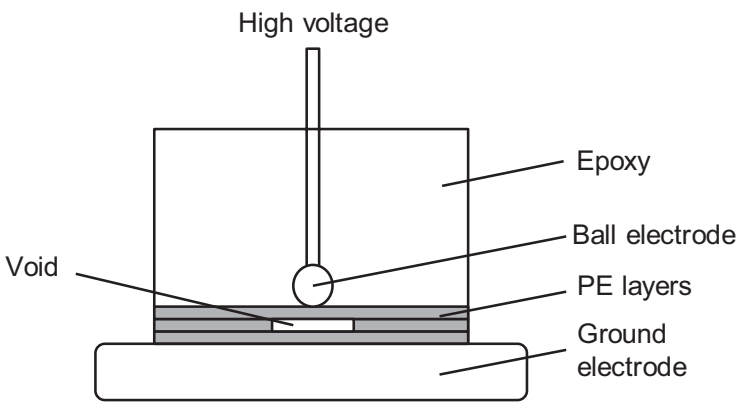

Fig. 1. Test object of the experiment [1]

\section{FEA MODEL GEOMETRY}

Fig. 2 shows a two-dimensional (2D) axial-symmetry model geometry that has been developed using a finite element analysis (FEA) software. The model dimensions are based on the actual test object that was used in the experiment. To model charge propagation along the void surface, the void surface is divided into 10 sections of equal surface area [4]. Table 1 shows the subdomain properties in the FEA model.

The partial differential equation (PDE) used to solve the electric field, $E$ in the model, where $\varepsilon$ is the permittivity is

$$
\vec{\nabla} \bullet \varepsilon \vec{E}=0
$$

\section{PD OCCURRENCE}

PD is assumed to occur only along the symmetry axis of the void $[5,6]$. Thus, only the electric field in the centre of the void, $E_{v}$ is checked for PD occurrence. When $E_{v}$ is larger than the inception field, $E_{i}$, the total electron generation rate (EGR), $G_{t}$ is calculated. $G_{t}$ equals to the summation of the EGR due to surface emission, $G_{s}$ and volume ionization, $G_{v}[7,8] . G_{s}$ is time dependent and calculated as

$$
G_{s}(t)=\alpha G_{s 0}\left|E_{v}\left(t_{P D}\right) / E_{i}\right| \exp \left[-\left(t-t_{P D}\right) / \tau_{d}\right] \exp \left|E_{v}(t) / E_{i}\right|
$$

where $G_{s 0}$ is the initial $G_{s}$ at $E_{i}, t_{P D}$ is the time of previous PD occurrence, $\tau_{d}$ is the effective charge decay time constant and $\alpha$ is the detrapping coefficient, depending on the polarity of surface charge during the electron detrapping. When electron detrapping is from positive charged surface, $\alpha=1$ while $\alpha<1$ when it is from negative charged surface.

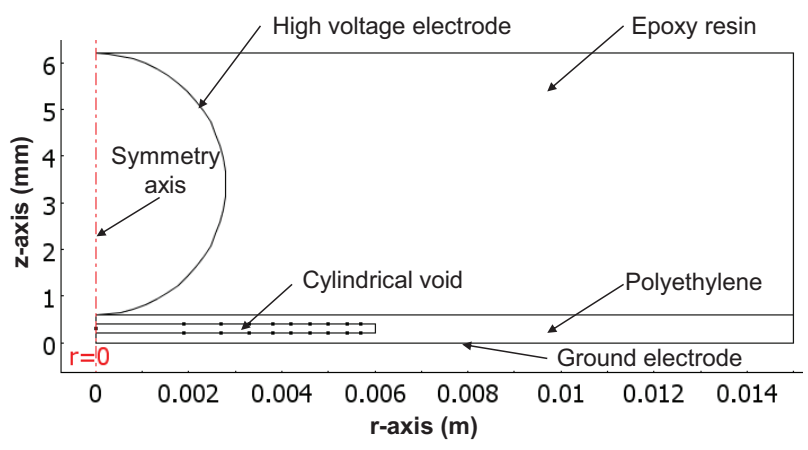

Fig. 2. 2D axial-symmetric FEA model geometry
TABLE I

Subdomain Parameters USED In THe SimUlation

\begin{tabular}{|c|c|}
\hline Subdomain & Value \\
\hline Polyethylene permittivity & 2.25 \\
\hline Epoxy resin permittivity & 4.4 \\
\hline Cylindrical void permittivity & 1 \\
\hline
\end{tabular}

After $G_{s}$ is calculated, $G_{t}(t)$ is obtained and the probability of a PD to occur is determined by

$$
P(t)=G_{t}(t) \Delta t
$$

where $\Delta t$ is the time stepping interval used in the simulation. $P(t)$ is then compared with a random number, $R$, where if $P(t)>R$, a PD will occur.

Once a PD occurs, charge density on one section of the void surface nearest to the axial symmetry is increased by $\rho$ [4]. When a PD occurs at positive $E_{v}$, the section of the void top surface is added with $-\rho$ while the section of the void bottom surface is added with $+\rho$. The opposite is done when a PD occurs at negative $E_{v}$.

In order to simplify the model, charges are assumed to propagate only up to the void surface section nearest to the symmetry axis. The charge propagation is also assumed symmetrical on top and bottom surfaces of the void. The electric field in the void centre, $E_{v}$ is checked whether it is less than the extinction field, $E_{x}$ or not. If $E_{v}$ is higher than $E_{x}$, another $-\rho$ and $+\rho$ are added on the void surface sections nearest to the symmetry axis. This process is repeated until $E_{v}$ is less than $E_{x}$.

The real PD charge magnitude is calculated by the total charge density added on the void surface during PD occurrence multiply with one section of the void surface area. The apparent charge magnitude is calculated by integration of charge density on the surface area of the grounded electrode. The effect of charge conduction along the void surface is neglected. Thus, charge distribution after a PD occurrence will remain the same until the next PD occurs.

\section{MEASUREMENT AND SimUlation RESUltS}

In order to match the simulation results in this work and the measurement results reported in [1], the parameters used in the simulation were chosen as in Table 2 . The inception field, $E_{i}$ was chosen based on the literature, the extinction field, $E_{x}$ was based on the measured minimum charge magnitude and $G_{v}$, $G_{s 0}, \alpha$ and $\rho$ were chosen through comparison between measured and simulated PRPD patterns. Figs. 3 and 4 show the measurement results that were obtained from the work in [1] and simulation results from this work. Both results are within reasonable agreement to each other.

TABLE II

Parameters USed in the Simulation

\begin{tabular}{|c|c|}
\hline Parameter & Value \\
\hline Applied voltage, $U_{a}$ & $3.5,4,5,6 \mathrm{kV}$ \\
\hline Applied frequency, $f$ & $200 \mathrm{~Hz}$ \\
\hline Time stepping interval, $\Delta t$ & $0.05 /(360 f) \mathrm{s}$ \\
\hline Simulation cycle & $100 \mathrm{cycles}^{-1}$ \\
\hline Inception field, $E_{i}$ & $5.5 \mathrm{kVmm}^{-1}$ \\
\hline
\end{tabular}




\begin{tabular}{|c|c|}
\hline Extinction field, $E_{x}$ & $4 \mathrm{kVmm}^{-1}$ \\
\hline EGR due to volume ionization, $G_{v}$ & $10 \mathrm{ks}^{-1}$ \\
\hline Initial EGR due to surface emission, $G_{s 0}$ & $30 \mathrm{ks}^{-1}$ \\
\hline Detrapping coefficient, $\alpha$ & 0.5 \\
\hline Effective charge decay time constant, $\tau_{d}$ & $2 \mathrm{~ms}$ \\
\hline Charge density increment, $\rho$ & $1 \times 10^{-4} \mathrm{Cm}^{-2}$ \\
\hline
\end{tabular}
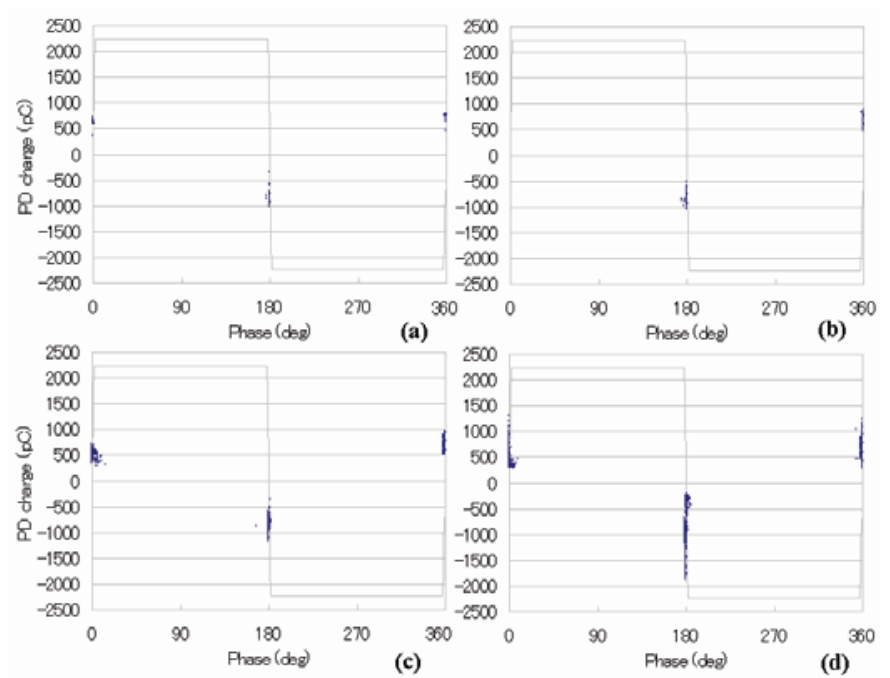

Fig. 3. PRPD patterns of the measurement results at; (a) $3.5 \mathrm{kV}$, (b) $4 \mathrm{kV}$, (c) $5 \mathrm{kV}$ and (d) $6 \mathrm{kV}[1]$

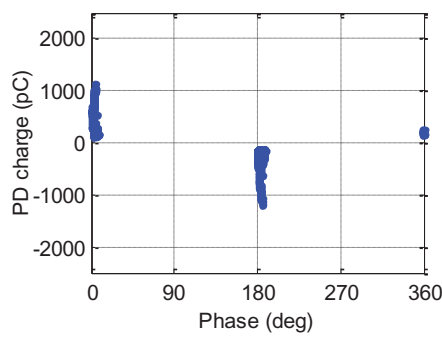

(a)



(c)

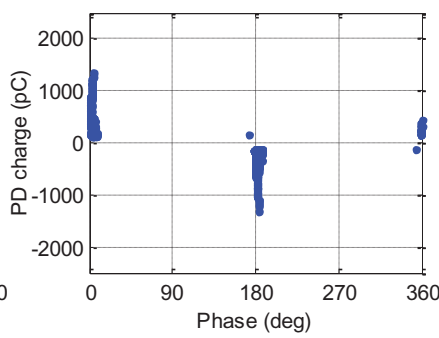

(b)

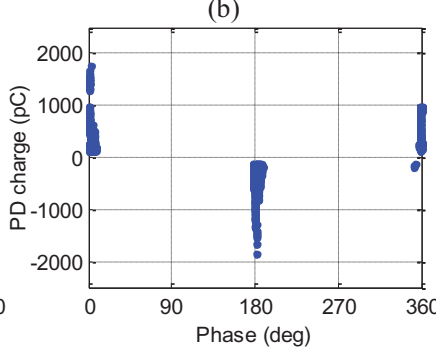

(d)
Fig. 4. PRPD patterns of the simulation results at; (a) $3.5 \mathrm{kV}$, (b) $4 \mathrm{kV}$, (c) 5 $\mathrm{kV}$ and (d) $6 \mathrm{kV}$

To understand how the PRPD patterns in Fig. 4 is obtained, the electric field magnitude against time of the first two cycles of $200 \mathrm{~Hz}, 3.5 \mathrm{kV}$ square waveform applied voltage is plotted, as shown in Fig. 5. The square waveform applied voltage is constructed by series of sine wave harmonics up to $25^{\text {th }}$ order. This is to ensure that PD can occur during the rising and falling edge of the applied voltage. If an ideal square waveform is used, PD will only occur at the peak of the applied voltage due to a very quick rise and fall of the voltage.

Referring to Fig. 5, at the beginning, the field in the void centre, $E_{v}$ equals to $E_{v 0}$, where $E_{v 0}$ is $E_{v}$ due to the applied field without any surface charge. Although $E_{v}$ has exceeded the inception voltage, $E_{i}$ during the rising edge of the applied voltage, PD does not occur due to unavailability of an initial free electron. At $0.5 \mathrm{~ms}$, the first PD occurs, resulting in $E_{v}$ to decrease until becomes less than the extinction field, $E_{x}$. The field due to surface charge, $E_{c}$ is in the opposite direction of $E_{v}$ when a PD occurs in the void. There is no PD occurs at all from $0.5 \mathrm{~ms}$ to $2.5 \mathrm{~ms}$ due to $E_{v}$ is less than $E_{i}$. This is because $E_{v}$ does not increase and remain constant.

At $2.5 \mathrm{~ms}$, when subsequent PD occurs on the rising edge of negative $E_{v}, E_{c}$ decreases. Since the direction of PD occurrence is opposite of the previous PD, new charges accumulated along the void surface neutralize the previously accumulated charges. $E_{c}$ then becomes positive and increases due to more charges of similar polarity accumulate along the void surface. Due to the time variation of electron availability when $E_{v}$ is larger than $E_{i}$, especially during the rising and falling edge of $E_{v}$, PDs occur at different magnitude of $E_{v}$. This results in variation of PD charge magnitude, as shown in Fig. 4. These sequences repeat in each cycle of the square waveform applied voltage.

Fig. 6 shows the electric field distribution in the FEA model before and after a PD occurs in the void at the peak of the applied voltage. Before a PD occurs (Fig. 6a), the electric field in the cylindrical void is higher than the polyethylene (PE) due to its permittivity is lower than PE. The electric field in the void is not uniform due to the ball electrode is used. The applied electric field from the ball electrode and towards the ground electrode is not uniform.

After the first PD occurs in the void, the electric field in the middle of the void decreases, as shown in Fig. 6b. This is due to charge density up to a certain value has been added along the void surface section nearest to the symmetry axis. A negative charge density was added on the void top surface while positive charge density was added on the void bottom surface. The electric field in the void can be seen heavily nonuniform after a PD occurs due to the influence of the electrode and charges along the void surface. In a real situation, the subsequent PDs are affected by the non-uniformity of the electric field in the void. Thus, the condition of each PD occurrence is different from each other. However, for simplicity of the model that has been developed in this work, PD is assumed to occur only from one end of the void surface, propagating across the void gap along the symmetry axis and reaches the opposite void surface.

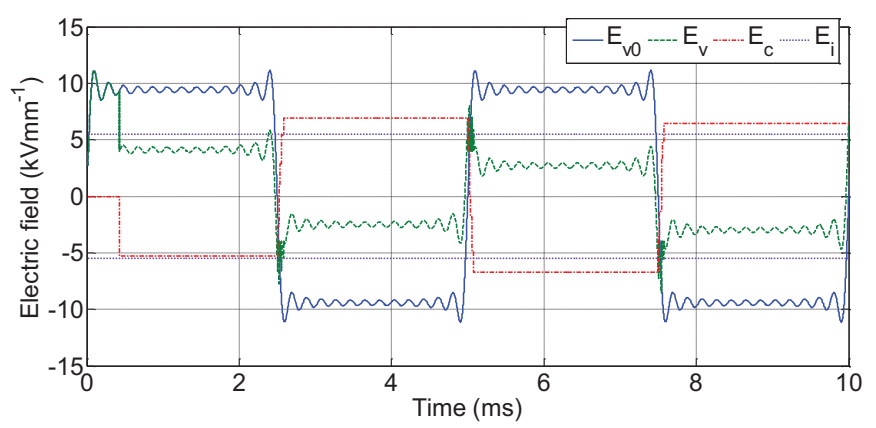

Fig. 5. Electric field magnitude against time of square waveform voltage 


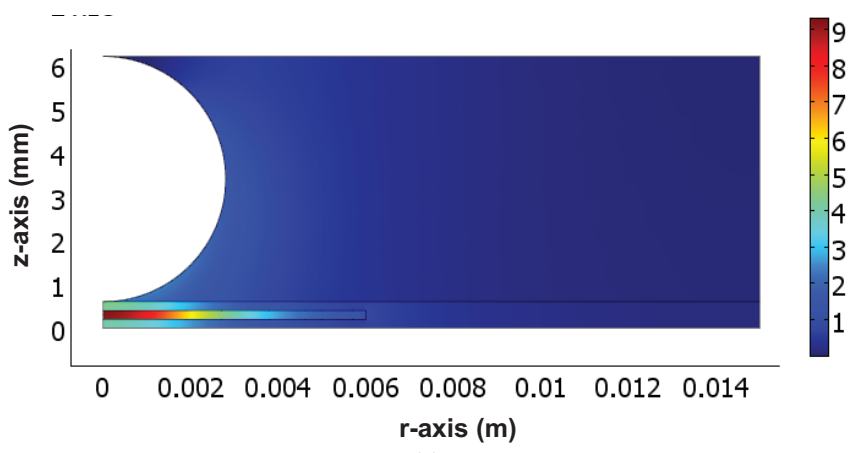

(a)

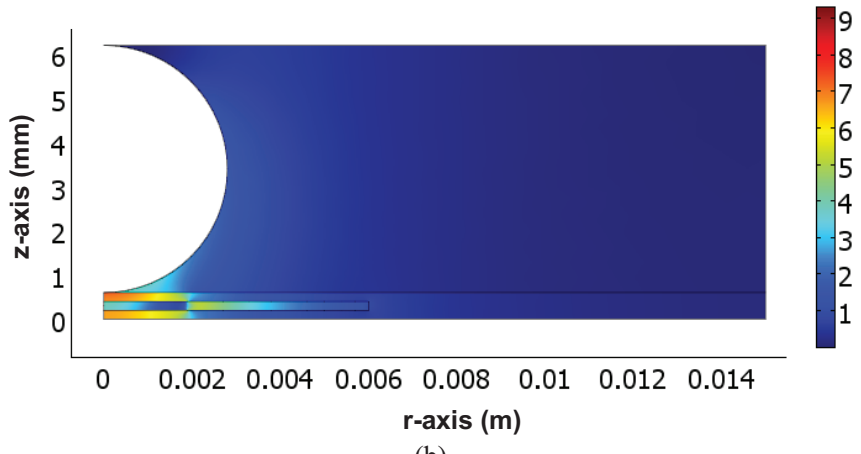

(b)

Fig. 6. Electric field distribution in the FEA model; (a) before and (b) after a PD occurs in the void.

\section{CONCLUSIONS}

A two-dimensional (2D) model of a cylindrical void in polyethylene dielectric material has been developed using finite element analysis (FEA) software. The model has been used to simulate partial discharge (PD) activity within the void when it is stressed with square waveform applied voltage. From comparison between the measurement results in literature and simulation results that were obtained in this work, both results agree to each other but with only slight difference. This is due to the over-simplified model used in this work, the unavailability of the actual data from the measurement results in literature and less precise square waveform applied voltage used. However, from the simulation model that has been developed, critical parameters affecting PD activity under square waveform applied voltage can be readily identified. These include the inception and extinction voltages and electron generation rate.

Future work will consider the actual measurement of PD activity by the authors and the results will be compared with the simulation results. Some improvements can also be performed on the current model. The model may consider the electric field dependent of charge propagation along the void surface during PD occurrence. For example, if the field along the propagation path is less than a certain limit, the charge propagation is terminated. Charge conduction along the void surface after a PD has occurred can also be considered. This may represent the change in the conductivity of the void surface due to aging process. A square waveform applied voltage which is near to ideal can be used to improve the model. However, this may increase the computational time since a smaller time stepping interval has to be used during the rising and falling edges of the applied voltage.

\section{ACKNOWLEDGMENT}

The author thanks the University of Malaya, Malaysia for supporting this work through the MOHE HIR grant (Grant no: H-16001-00-D000048).

\section{REFERENCES}

[1] W. Kai, T. Okamoto, and Y. Suzuoki, "Effects of discharge area and surface conductivity on partial discharge behavior in voids under square voltages," IEEE Transactions on Dielectrics and Electrical Insulation, vol. 14, pp. 461-470, 2007.

[2] E. Lindell, T. Bengtsson, J. Blennow, and S. M. Gubanski, "Influence of rise time on partial discharge extinction voltage at semi-square voltage waveforms," IEEE Transactions on Dielectrics and Electrical Insulation, vol. 17, pp. 141-148, 2010.

[3] B. Florkowska, M. Florkowski, J. Roehrich, and P. Zydron, "Partial discharge mechanism in a non-uniform electric field at higher pressure," IET Science, Measurement \& Technology, vol. 5, pp. 59-66, 2011.

[4] H. Illias, G. Chen, and P. L. Lewin, "The influence of spherical cavity surface charge distribution on the sequence of partial discharge events," Journal of Physics D: Applied Physics, vol. 44, pp. 1-15, 2011.

[5] H. Illias, G. Chen, and P. L. Lewin, "Partial Discharge Behavior within a Spherical Cavity in a Solid Dielectric Material as a Function of Frequency and Amplitude of the Applied Voltage," IEEE Transactions on Dielectrics and Electrical Insulation, vol. 18, pp. 432-443, 2011.

[6] H. Illias, G. Chen, and P. L. Lewin, "Partial discharge within a spherical cavity in a dielectric material as a function of cavity size and material temperature,"IET Science, Measurement \& Technology, vol. 6, pp. 5262, 2012.

[7] F. Gutfleisch and L. Niemeyer, "Measurement and simulation of PD in epoxy voids," IEEE Transactions on Dielectrics and Electrical Insulation, vol. 2, pp. 729-743, 1995.

[8] L. Niemeyer, "A generalized approach to partial discharge modeling," IEEE Transactions on Dielectrics and Electrical Insulation, vol. 2, pp. 510-528, 1995. 